\title{
Radiomics in evaluation of small-hepatocellular carcinoma and benign cirrhotic nodule based on magnetic resonance plain images
}

\section{XiaoNing Gao (D748117050@qq.com)}

the second hospital of dalian medical university

\section{Kun Guo}

the second hospital of dalian medical university

JiaWen Luo

the second hospital of dalian medical university

Jie Bian

the second hospital of dalian medical university

Technical advance

Keywords: Radiomics, s-HCC, MRI

Posted Date: August 4th, 2020

DOI: https://doi.org/10.21203/rs.3.rs-45433/v1

License: (c) (1) This work is licensed under a Creative Commons Attribution 4.0 International License.

Read Full License 


\section{Abstract}

Background There are adequate evidences showing that early diagnosis significantly improves the prognosis of patients. Currently, radiology diagnosis to s-HCC is still difficult. Radiomics, a new type of quantitative imaging diagnosis method, has been widely used in the study of multi-system diseases. The purpose of this study is to evaluate the value of radiomics based on MRI plain images in differentiating sHCC and benign cirrhotic nodule.

Methods A total of 78 patients with 114 lesions ( 89 s-HCCs and 25 benign cirrhotic nodules) were retrospectively enrolled during September 2017 to January 2020. MRI plain images (T1WI, T2WI) of each nodule was segmented to form $\mathrm{VOI}$, and 1223 quantitative radiomics features were extracted from each sequence. 10 optimal features were respectively selected from the T1WI, T2WI, and T1WI+T2WI based on SelectKBest. Logistic Regression (LR) was applied in establishing 2 radiomics models based on single sequence images (T1WI and T2WI) respectively and 1 radiomics model based on $\mathrm{T} 1 \mathrm{Wl}+\mathrm{T} 2 \mathrm{Wl}$. Clinical information (including age, gender, AFP level and the longest diameter of lesion) was collected and analyzed using binary logic analysis to obtain statistically significant $(p<0.05)$ clinical features. Combining the optimal radiomics features based on T1WI+T2WI and significant clinical features, the fusion model was established using LR and was expressed in nomogram. The AUC, sensitivity and accuracy of the 4 models were obtained. Delong test was used to compare the AUC of the 4 models.

Results 78 patients with 114 lesions were included in this study. There were 89 s-HCCs, 11 of nodules were e-HCCs, and the remaining 25 were benign cirrhotic nodules, including 3 DNs and 22 RNs. There was no significant difference in diagnostic performance among the radiomics model based on T2WI, $\mathrm{T} 1 \mathrm{WI}+\mathrm{T} 2 \mathrm{WI}$ and the fusion model. And their diagnostic performance were higher than that of the radiomics model based on T1WI.

Conclusions The radiomics model based on MRI plain images can be used to differentiate s-HCC and benign cirrhotic nodule. Radiomics features obtained from T2WI played a key role in the differentiation of s-HCC and benign cirrhotic nodule.

\section{Background}

Hepatocellular carcinoma (HCC) is the second cause of cancer-related death worldwide ${ }^{[1]}$. Since most HCCs are diagnosed in the middle and advanced stages of the disease, so that effective clinical treatment is unavailable, the median survival of less than 1 year and a 5 -year survival rate of less than $10 \%{ }^{[2]}$. Some clinical studies have shown that surgical intervention for s-HCC can reduce the recurrence rate and improve the cure rate of HCC patients ${ }^{[3-5]}$. Therefore, increasing the diagnostic accuracy of sHCC is of great significance for improving the prognosis of patients.

At present, imaging has become a non-invasive and accurate method for detection and diagnosis of HCC. Enhanced MRI provide significantly higher detection rate and diagnosis performance of HCC, even s-HCC, when combine with typical enhancement characteristics of HCC and hepatobiliary images obtained by 
hepatobiliary agents ${ }^{[6,7]}$. However, due to the incomplete angiogenesis, the manifestation of the enhancement performance of s-HCC can be atypical. Takayasu et al have found that $24 \%$ of the s-HCC between $1.1 \mathrm{~cm}$ and $2 \mathrm{~cm}$ in diameter can be hypoenhancement ${ }^{[8]}$. And some other studies have also pointed out that $27 \%-34 \%$ of s-HCC presented poor blood supply ${ }^{[9]}$. The application of hepatobiliary contrast agents is more likely to produce respiratory artifacts, which affects the image quality of late arterial phase. Moreover, as the uptake of hepatobiliary contrast agent is closely related to the liver function of patients, it can have a great impact on the images ${ }^{[10]}$. So, there are certain deficiencies in differentiating s-HCC and benign cirrhotic nodule based only on qualitative imaging features. In addition, as high-risk patients of HCC may need repeated imaging examinations during follow-up, lots of enhancement examinations will increase the cost of patients. And some patients cannot undergo enhancement examinations due to contraindications. MRI plain images is more available and can be of great use.

According to the clinical diagnosis guidelines, pathological results of high-risk patients of HCC with indeterminate nodules can be obtained by puncture biopsy. Because the sample of lesion tissue obtained by puncture is insufficient, especially cirrhotic-related nodule with strong heterogeneity, diagnostic accuracy of biopsies is relatively low. Also the procedure may cause some side effects such as tumor dissemination and bleeding in puncture tract [10]. We urgently need a more efficient and accurate noninvasive method to improve the ability to distinguish between s-HCC and benign cirrhotic nodule, so as to increase the prognosis of patients with $\mathrm{HCC}^{[11-13]}$.

In 2012, Lambin ${ }^{[14]}$ has proposed a new image quantitative analysis tool -- radiomics. By extracting and selecting quantitative imaging features, robust features can be obtained to establish objective and accurate connection between imaging and biological information. Nowadays, radiomics has been widely used in the study of inflammatory or neoplastic lesions in the brain, breast, soft tissue, and etc. In terms of liver, the researches mainly focus on the identification of benign and malignant lesions, the prediction of HCC microvascular infiltration, and the evaluation of postoperative efficacy of HCC. So far, there are few studies involving in differential diagnosis of s-HCC and benign cirrhotic nodule by radiomics.

The purpose of this study was to evaluate the value of radiomics based on MRI plain images in identifying s-HCC and benign cirrhotic nodule.

\section{Methods}

\section{Study population}

Patients with cirrhosis who underwent liver transplantation and hepatectomy in our hospital from January 2017 to January 2020 were retrospectively included. MRI plain and enhanced images were obtained by using hepatobiliary agents or extracellular contrast agents within 4 weeks before surgery. Ensure the consistency of all machines and scanning parameters. Enrolled patients met the inclusion criteria: 邓the longest diameter of the lesion shown by imaging was $\leq 2 \mathrm{~cm}$; 『pathological diagnosis of the 
nodules were cirrhotic - related nodules. The exclusion criteria were as follows: 『patients underwent any preoperative intervention treatment; \the lesion was not clearly shown on MRI plain images; 》the gross specimen did not correspond to the location and size of the nodules shown in the imaging. The study population was randomly divided into training group and test group at a ratio of 6:4.

\section{MRI protocol}

All enrolled patients were examined with 3.0T MRI (Verio; Siemens, Erlangen, Germany), with 8-channel body phased array coil and turbo spin-echo (TSE) sequence acquisition collection under respiratory trigger. Abdominal pressure band was applied to the lower abdomen of the patient to reduce artifacts caused by abdominal respiratory movements. The scanning sequence of MRI included: axial T1WI and axial fat-suppression T2WI. The parameters of each MRI sequence are shown in Table 1.

\section{Image-pathology comparison method}

Refer to the MRI images, the liver transplantation specimens or resected specimens were cut at an interval of nearly $1 \mathrm{~cm}$. Pathological specimens of the abnormal signal nodules shown in the images were corresponding to the dissected liver specimen. The pathological specimens were sectioned, dehydrated and paraffin-embedded, stained with hematoxylin-eosin, and then subjected to histological and immunohistochemical examination. The image-pathology comparison method was illustrated in Fig. 1 and 2. The pathological diagnosis of liver lesions was evaluated according to the International Consensus Group for Hepatocellular Neoplasia criteria.

\section{Model establishment and comparison}

\subsection{Establishment of radiomics model}

\subsubsection{Segmentation}

MRI plain images were transferred to the Medical Standard - Darwin Intelligent Scientific Research Platform. A radiologist segmented the lesions on the T1WI and T2WI to form a three-dimensional VOI; and two senior radiologists with more than 10-year experience in abdominal imaging diagnosis proofread the edge of the lesions. In case of disagreement, the lesions would be segmented again after 2 weeks (Fig. 3). All three physicians were blinded to pathological results, but were aware of the purpose and method of the study.

\subsubsection{Extraction and selection}

A total of 1223 radiomics features were extracted from the segmented images at each plain sequence, which mainly include first-order features, 2D and 3D shape features, texture features (including gray-level co-occurrence matrix (GLCM) features, gray-level run-length matrix (GLRLM) features, gray-level size zone matrix (GLSZM) features, gray-level dependence matrix (GLDM) features, and neighbourhood gray-tone 
difference matrix (NGTDM) features). To avoid overfitting, the dimension reduction method of SelectKBest was applied to select the optimal radiomics features.

\subsubsection{Establishment of radiomics model}

LR was applied to establish three groups of radiomics models using the 10 optimal radiomics feature selected from $\mathrm{T} 1 \mathrm{WI}, \mathrm{T} 2 \mathrm{WI}$, and $\mathrm{T} 1 \mathrm{WI}+\mathrm{T} 2 \mathrm{WI}$ images, respectively. Radiomics models were evaluated using the AUC.

\subsection{Establishment of fusion model}

\subsubsection{Collection of clinical feature}

A radiologist collected clinical information of each patient, including age, gender, AFP level, and the longest diameter of lesion. The radiologist was blinded to pathological results.

\subsubsection{Establishment of fusion Model}

The clinical features with statistical significance $(p<0.05)$ was obtained using binary logic analysis method. LR was applied to establish a fusion model, based on optimal radiomics features obtained by T1WI+T2WI and significant clinical features, and expressed in nomogram. The AUC were used to evaluate the fusion model.

\subsection{Comparison}

Delong test was used to compare the AUCs of $\mathrm{T} 1 \mathrm{WI}, \mathrm{T} 2 \mathrm{WI}, \mathrm{T} 1 \mathrm{WI}+\mathrm{T} 2 \mathrm{WI}$ radiomics models and fusion model.

\section{Statistic analysis}

Statistical analysis were carried out using Medcale statistical software (http://www.medcalc.org). Binary logistic regression analysis was used to select clinical features with a p value less than 0.05 . Radiomics feature extraction, dimension reduction and model establishment were obtained from the Medical Standard - Darwin Intelligent Scientific Research Platform (Beijing, Yizhun Medical Al Co., Ltd).

\section{Results}

\section{Characteristics of patients}

Initially, a total of 82 patients were enrolled, 4 patients were excluded due to severe ascites ; finally 78 patients with 114 lesions were included in this study. 70 patients had chronic hepatitis B cirrhosis, 3 patients had chronic hepatitis $\mathrm{C}$ cirrhosis, 3 patients had autoimmune cirrhosis, and 2 patients had alcoholic cirrhosis. There were 89 s-HCC, 11 of nodules were e-HCCs, and the remaining 25 were benign cirrhotic nodules, including 3 DNs and 22 RNs. Patients' detail information are shown in Table 2. 


\section{Radiomics models}

The flow chart for the establishment of radiomics model is shown in Fig. 3. The optimal radiomics features obtained based on T1WI images are shown in Fig. 1 in supplementary, the radiomics scores (Rad score) are shown in Formula 1. The AUC, sensitivity and specificity of the training group were 0.757 (95\% Cl $0.638-0.853), 83.02 \%$ and $66.67 \%$, respectively. The AUC, sensitivity and specificity of the test group were 0.789 (95\% $\mathrm{Cl} 0.643-0.895), 88.89 \%$ and $80.00 \%$,respectively. The receiver operating characteristic curves (ROCs) of the models are shown in Fig. 4.

RadScore $=-1.533^{\star} T 1$ WI_wavelet-LHL_glcm_InverseVariance+

1.083*T1WI_wavelet-HLL_firstorder_Mean+

0.851*T1WI_wavelet-HLL_firstorder_Median+

0.558*T1WI_wavelet-HLL_firstorder_Minimum-

$0.525^{\star}$ T1WI_wavelet-LHL_firstorder_InterquartileRange-

0.477*T1WI_wavelet-HLH_glcm_Autocorrelation-

0.460*T1WI_wavelet-HLH_gldm_LargeDependenceHighGrayLevelEmphasis-

0.460*T1WI_wavelet-HLH_gldm_HighGrayLevelEmphasis-

0.187*T1WI_wavelet-HHL_firstorder_InterquartileRange-

0.049*T1WI_wavelet-HHL_firstorder_90Percentile-0.688

\section{Formula (1)}

The optimal radiomics features obtained based on T2WI images are shown in Fig. 2 in supplementary, and the radiomics score are shown in Formula 2. The AUC, sensitivity and specificity of the training group were 0.903 (95\% Cl $0.807-0.962), 86.79 \%$ and $86.67 \%$, respectively. The AUC, sensitivity and specificity of the test group were $0.778(95 \% \mathrm{Cl} 0.632-0.887), 75.00 \%$, and $80.00 \%$, respectively. The ROCs of the models are shown in Fig. 5.

RadScore $=-1.584 *$ T2WI_wavelet-HLL_firstorder_Mean-

1.512*T2WI_wavelet-LHL_firstorder_Median-

1.487*T2WI_wavelet-HLL_ngtdm_Contrast-

1.318*T2WI_wavelet-LHL_firstorder_Mean-

0.959*T2WI_square_gldm_DependenceNonUniformityNormalized-

0.618*T2WI_square_gldm_LargeDependenceLowGrayLevelEmphasis- 
0.414*T2WI_square_glcm_MaximumProbability-

$0.279 * T 2 W I$ square_glcm_JointEnergy-

0.156*T2WI_square_glszm_SizeZoneNonUniformityNormalized+3.391 Formula (2)

The optimal radiomics features obtained based on T1WI+T2WI are shown in Fig. 3 in supplementary, and the radiomics score is shown in Formula 3 . The AUC, sensitivity and specificity of the training group were 0.926 (95\%, Cl $0.836-0.975), 98.11 \%$ and $86.67 \%$, respectively. The AUC, sensitivity and specificity of the test group were 0.828 (95\% $\mathrm{Cl} 0.688-0.923), 58.33 \%$ and $100.00 \%$, respectively. The ROCs of the models are shown in Fig. 6.

RadScore $=-1.433^{\star}$ T2WI_wavelet-HLL_firstorder_Mean+

$1.303^{*}$ T1WI_wavelet-HLL_firstorder_Minimum-

1.299*T2WI_wavelet-HLL_ngtdm_Contrast-

1.291*T2WI_wavelet-LHL_firstorder_Median-

1.287*T2WI_wavelet-LHL_firstorder_Mean-

1.108*T2WI_square_gldm_DependenceNonUniformityNormalized-

$0.883^{*}$ T2WI_square_glcm_JointEnergy-

0.598*T1WI_wavelet-HHL_firstorder_InterquartileRange-

0.229*T2WI_wavelet-HLL_firstorder_Median+

0.186*T1WI_wavelet-HLL_firstorder_Median+1.989

Formula (3)

\section{Fusion model and nomogram}

Binary logistic regression analysis showed that the patient's gender was statistically significant in differentiating between $\mathrm{s}-\mathrm{HCC}$ and benign cirrhotic nodule $(p=0.023, p<0.05)$, and the remaining clinical features were not statistically significant. The nomogram of the diagnostic model obtained combined with clinical characteristics and optimal radiomics features by applying logistic regression is shown in Fig. 7. The AUC, sensitivity and specificity of the training group were 0.935 (95\% $\mathrm{Cl} 0.847-0.980), 94.34 \%$ and $93.33 \%$, respectively. The AUC, sensitivity and specificity of the test group were $0.772(95 \% \mathrm{Cl} 0.625-$ $0.883), 97.22 \%$ and $60.00 \%$, respectively. The ROCs of the models are shown in Fig. 8.

\section{Comparison of diagnostic performance}


Delong test has proved that, the diagnositic performance had no difference among radiomics model based on T2WI, T1 WI+T2WI and fusion model ( $\mathrm{p} \otimes 0.05)$. The diagnositic performances of training group of these three models were higher than that of radiomics model based on T1WI $(p=0.0379, p=0.0213, p$ $=0.008)$.

\section{Discussion}

This study verified that the application of radiomics based on $\mathrm{T} 1 \mathrm{WI}$ and $\mathrm{T} 2 \mathrm{WI}$ can be used to differentiate s-HCC and benign cirrhotic nodule. In the radiomics model based on a single sequence, we found the radiomics features obtained from T2WI play a key role in differentiating between s-HCC and benign cirrhosis nodule. We considers this has to do with the advantages of T2WI sequence. T2WI sequence can better reflect the metabolism, blood supply and interstitial structure of lesions by showing the content of $1 \mathrm{H}$. First, the rapid growth and proliferation of tumor cells, causing the increase of intracellular water molecules and phospholipid of the cell membrane, results that the content of $1 \mathrm{H}$ in s-HCC is significantly higher than that of benign cirrhotic nodule [15], which can be reflected on T2WI. Second, the growth of tumor cells can induce and promote tumor angiogenesis $₫$ which in turn can facilitate the proliferation of tumor cells. Pathological studies have shown that there is little unpaired arteries and sinusoidal capillarization in RN and low grade dysplastic nodule $\mathrm{LG}$ GNQ. While in high grade dysplastic nodule (HGDN), e-HCC, s-HCC, the unpaired arteries gradually increase with the growth of lesion diameter [16-18]. Hidenori et al indicated that the content of tumor vessels in e-HCC was significantly higher than that of HGDN [19]. In summary, the heterogeneity of tumor cells and interstitial tissue structure in the lesion may cause differences of T2WI signal between s-HCC and benign cirrhotic nodule. Moreover, hypoxia caused by incomplete angiogenesis resulting in the occurrence of iron deposits in the benign cirrhotic nodule may affect the T2WI signal of lesion [20-22]. This study indicated that there was no statistically significant difference in diagnostic performance between the radiomics model based on T1WI+T2WI and T2WI single-sequence $(p>0.05)$, but the diagnostic performance of the two models was higher than that of the radiomics model based on T1WI single-sequence T1WI. The optimal radiomics features obtained by T1WI and T2WI through SelectKBest mainly include some first-order features (including average value, median, and minimum in VOI) and texture features (including JointEnergy in GLCM, Dependence NonUniformity Normalized in GLDM, and Contrast in NGTDM). These texture features mainly reflect the heterogeneity of lesion images [23]. According to the radiomics score obtained in this study, the conclusion is that s-HCC has more significant heterogeneity than benign cirrhotic nodule. We consider that this is related to the heterogeneity of tumor cells and interstitial structures in s-HCC. As mentioned above, tumor cells in s-HCC divided and proliferated vigorously. Different genes or other macromolecule changes may appear in the daughter cells generated by multiple generations of division and proliferation, which lead to greater heterogeneity between tumor cells. There are also abundant unpaired arteries and hepatic sinusoidal tumor vessels in the tumor interstitial structure. In addition, the fibrous septa exists between the HCC sub-nodules or between the necrotic areas and HCC tissue [24], resulting in considerable heterogeneity in the interstitial structure of the lesion. The fusion model was established based on the combination of optimal radiomics features based on $\mathrm{T} 1 \mathrm{Wl}+\mathrm{T} 2 \mathrm{Wl}$ and optimal clinical features. We 
expressed the fusion model in nomogram intuitively, which is more convenient for clinicians and patients to understand. The results showed that there was no statistically difference between diagnostic performance of the fusion model, radiomics model based on T1WI+T2WI and T2WI single-sequence. In the collected clinical features, gender had certain significance for diagnosis of HCC. This reminds male patients to be more alert to the occurrence of s-HCC. AFP is the most common and readily available tumor marker of HCC in clinical studies. Clinical researchs have shown that AFP plays an important role in predicting microvascular invasion, $\mathrm{Ki}-67$ and prognosis of HCC. Therefore, this study included AFP as a clinical diagnostic factor. However, according to the statistical analysis, AFP had no considerable significance in differentiating s-HCC from benign cirrhotic nodule. Malaguarnera et al demonstrated that $80 \%$ of s-HCC did not show an increase in AFP levels [25]. Johnson et al also pointed out that when the diameter of the tumor is small in early stage of HCC, AFP level cannot be used for the diagnosis of HCC, which is likely to cause missed diagnosis[26]. The results of this study are consistent with the conclusions of the above experts and scholars. Due to the large heterogeneity within cirrhotic-related nodule, the most critical step in the study is to obtain accurate pathological results of nodules. In previous studies, most lesions are obtained by needle biopsy or follow-up observation. In this study, all pathological results were obtained from patients who had underwent liver transplantation or hepatectomy. Moreover, radiomics, the method of quantitative analysis of images, can obtain more objective, accurate, stable radiomics features and models, which have high clinical application value. However, there are still some limitations of this study. First, the data in this study were all obtained from our hospital. The amount of date was not sufficient, and the classification of data was unbalanced. Thereby, we still need a large number of external data to verify the radiomics model to obtain more stable radiomics features and diagnostic model. Second, this study did not make more detailed classification of benign cirrhotic nodule of which malignant transformation rates are different. Clinical studies by Kobayashi et al. showed that the 1-, 3-, and 5-year malignant transformation rates of HGDN were $46.2 \%, 61.5 \%$ and $80.8 \%$, respectively, which were significantly higher than those of $\operatorname{LGDN}(2.6 \%, 30.2 \%, 32.6 \%)$ and large RN $(3.3 \%, 9.7 \%, 12.4 \%)[27]$. Thus, if benign cirrhotic nodules are classified, a meaningful radiomics model can be generated to facilitate clinical close monitoring of lesions with higher malignant transformation rate.

\section{Conclusions}

The radiomics model based on MRI plain images can be used to differentiate s-HCC and benign cirrhotic nodule. Radiomics features obtained from T2WI played a key role in the differentiation of s-HCC and benign cirrhotic nodule.

\section{Abbreviations}

AFP Alpha-fetoprotain

AUC Area under receiver operating characteristic curve

DNs Dysplastic nodules 
e-HCCs early-Hepatocellular carcinomas

GLCM Gray-level co-occurrence matrix

GLDM Gray-level dependence matrix

HCC Hepatocellular carcinoma

HGDN High grade dysplastic nodule

LGDN Low grade dysplastic nodule

LR Logistic regression

MRI Magnetic resonance imaging

NGTDM Neighbourhood gray-tone difference matrix

Rad score Radiomics score

RNs Regenerative nodules

ROC Receiver operating characteristic curve

s-HCC small-Hepatocellular carcinoma

TSE Turbo spin-echo

VOI volume of interest

\section{Declarations}

Ethical approval and consent to participate: The institutional ethics committee of the Second Hospital of Dalian Medical University have approved this retrospective study and waived patient informed consent. Consent to publish: Not Applicable. Availability of data and materials: The supporting date are availability from the corresponding author. Competing interests: The Authors declared that they had no conflicts of interests in their authorship and publication of this Contribution. Funding: Not Applicable. Authors' contributions: KG, JWL and XNG conceived and designed research; KG and XNG collected data and conducted research; XNG wrote the initial paper; KG, JWL and JB revised the paper; XNG had primary responsibility for final content. All authors read and approved the final manuscript. Acknowledgements: The authors will thank the Medical Standard - Darwin Intelligent Scientific Research Platform for the great help in technical support》 All supporting data can be accessed by contacting corresponding Author.

\section{References}


[1] Trevisani F, Cantarini MC, Wands JR, et al. Recent advances in the natural history of hepatocellular carcinoma. Carcinogenesis 2008; 29 (7): 1299-1305.

[2] McGlynn KA, London WT. Epidemiology and natural history of hepatocellular carcinoma. Best Pract Res Clin Gastroenterol 2005;19 (1): 3-23.

[3] Takayama T, Makuuchi M, Hirohashi S, et al. Early hepatocellular carcinoma as an entity with a high rate of surgical cure. Hepatology 1998; 28:1241- 46.

[4] Midorikawa Y, Yamamoto S, Tsuji S, et al. Allelic imbalances and homozygous deletion on 8p23.2 for stepwise progression of hepatocarcinogenesis. Hepatology 2009;49:513-22.

[5] Yamamoto M, Takasaki K, Otsubo T, et al. Favorable surgical outcomes in patients with early hepatocellular carcinoma. Ann Surg 2004; 239:395-9.

[6] Singal A, Volk ML, Waljee A, Salgia R, Higgins P, Rogers MA, et al. Meta-analysis: surveillance with ultrasound for earlystage hepatocellular carcinoma in patients with cirrhosis. Aliment Pharmacol Ther 2009;30:37-47

[7] Yu NC, Chaudhari V, Raman SS, Lassman C, Tong MJ, Busuttil RW, et al. CT and MRI improve detection of hepatocellular carcinoma, compared with ultrasound alone, in patients with cirrhosis. Clin Gastroenterol Hepatol 2011;9:161-167

[8] Takayasu K, Arii S, Sakamoto M, et al. Clinical implication of hypovascular hepatocellular carcinoma studied in 4,474 patients with solitary tumour equal or less than 3cm[J]. Liver International, 2013, 33(5):762-770.

[9] Bolondi L, Gaiani S, Celli N, et al. Characterization of small nodules in cirrhosis by assessment of vascularity: the problem of hypovascular hepatocellular carcinoma. Hepatology. 2005; 42 (1): 27-34.

[10]Min JH, Kim YK, Kang TW, et al. Artifacts during the arterial phase of gadoxetate disodium-enhanced MRI: Multiple arterial phases using view-sharing from two different vendors versus single arterial phase imaging. Eur Radiol, 2018, 28(8): 3335-3346.

[10] Silva M A, Hegab B, Hyde C, et al. Needle track seeding following biopsy of liver lesions in the diagnosis of hepatocellular cancer: a systematic review and meta-analysis[J]. Gut, 2008, 57 (11): 15921596

[11] Takayama T, Makuuchi M, Hirohashi S, et al. Early hepatocellular carcinoma as an entity with a high rate of surgical cure. Hepatology 1998; 28:1241-46. 
[12] Midorikawa Y, Yamamoto S, Tsuji S, et al. Allelic imbalances and homozygous deletion on 8p23.2 for stepwise progression of hepatocarcinogenesis. Hepatology 2009; 49: 513-22.

[13] Yamamoto $M$, Takasaki K, Otsubo T, et al. Favorable surgical outcomes in patients with early hepatocellular carcinoma. Ann Surg 2004; 239: 395-9.

[14] Lambin P, Rios-Velazquez E, Leijenaar R, et al. Radiomics: Extracting more information from medical images using advanced feature analysis[J]. European journal of cancer (Oxford, England: 1990), 2012, 48 (4): 441-446.

[15] Shen ZW, Cao Z, You KZ, et al. Quantification of choline concentration following liver cell apoptosis using 1H magnetic resonance spectroscopy. World J Gastroenterol. 2012; 18: 1130-1136.

[16] Palmer WC, Patel T. Are common factors involved in the pathogenesis of primary liver cancers? A meta-analysis of risk factors for intrahepatic cholangiocarcinoma. J Hepatol 2012; 57 (1): 69-76.

[17] Coleman WB. Mechanisms of human hepatocarcinogenesis. Curr Mol Med 2003;3(6):573-588.

[18] Kitao A, Matsui O, Yoneda N, et al. The uptake transporter OATP8 expression decreases during multistep hepatocarcinogenesis: correlation with gadoxetic acid enhanced MR imaging. Eur Radiol 2011;21(10):2056-2066.

[19] Ojima H, Masugi $Y$, Tsujikawa $\mathrm{H}$, et al. Early hepatocellular carcinoma with high-grade atypia in small vaguely nodular lesions[J]. Cancer Science, 2016, 107(4):543-550.

[20] International Consensus Group for Hepatocellular Neoplasia. Pathologic diagnosis of early hepatocellular carcinoma: a report of the international consensus group for hepatocellular neoplasia. Hepatology 2009;49(2):658-664.

[21] Terada T, Kadoya M, Nakanuma Y, Matsui O. Iron-accumulating adenomatous hyperplastic nodule with malignant foci in the cirrhotic liver. Histopathologic, quantitative iron, and magnetic resonance imaging in vitro studies. Cancer 1990;65(9):1994-2000.

[22] Gurusamy K. Trace element concentration in primary liver cancers-a systematic review. Biol Trace Elem Res 2007;118(3):191-206.

[23] Zwanenburg, A., Leger, S., Vallières, M., and Löck, S. (2016). Image biomarker standardisation initiative - feature definitions. In eprint arXiv:1612.07003 [cs.CV]

[24] Ishizaki M, Ashida K, Higashi T, et al. The formation of capsule and septum in human hepatocellular carcinoma. Virchows Arch 2001;438(6):574-580.

[25] Malaguarnera G, Giordano M, Paladinal I, et al. Serum markers of hepatocellular carcinoma. Dig Dis Sci, 2010, 55: 2744-2755. 
[26] Johnson, Philip J. The Role of Serum Alpha-Fetoprotein estimation in the Diagnosis and Management of Hepatocellular Carcinoma[J]. Clinics in Liver Disease, 2001, 5(1):145-159.

[27] Kobayashi M, Ikeda K, Hosaka T, et al. Dysplastic nodules frequently develop into hepatocellular carcinoma in patients with chronic viral hepatitis and cirrhosis. Cancer 2006; 106: 636-47.

\section{Tables}

Table 1. MRI conventional sequence parameter

\begin{tabular}{lcrcccc}
\multicolumn{2}{r}{ TR $₫ \mathrm{~ms} \rrbracket$} & $\mathrm{TE}(\mathrm{ms})$ & Matrix & thickness $(\mathrm{mm})$ & slice gap $(\mathrm{mm})$ & NEX \\
\hline Axial T1WI & 200 & 2.2 & $256^{\prime} 160$ & 5 & 0 & 1 \\
\hline Axial T2WI & infinite & 8.5 & $288^{\prime} 256$ & 5 & 1 & 2
\end{tabular}

Table 2. patients' information

\begin{tabular}{|c|c|c|c|c|c|c|c|}
\hline & \multicolumn{3}{|c|}{ Training group $(n=68)$} & \multicolumn{3}{|c|}{ Test group $(n=46)$} & \multirow[t]{2}{*}{$P$ value } \\
\hline & $\mathrm{HCC}$ & benign & $P$ value & $\mathrm{HCC}$ & benign & $P$ value & \\
\hline Age-ranges & $35-79$ & $32-65$ & 0.546 & 59.5 & 63.5 & 0.588 & 0.417 \\
\hline gender & & & 0.02 & & & 0.575 & 0.023 \\
\hline male & 45 & 8 & & 25 & 5 & & \\
\hline female & 8 & 7 & & 11 & 5 & & \\
\hline size(mm), median & 15 & 15 & 0.276 & 15 & 17 & 0.327 & 0.272 \\
\hline AFP(IU/ml), median & 5.07 & 2.9 & 0.608 & 7.4 & 2.6 & 0.488 & 0.468 \\
\hline
\end{tabular}

\section{Figures}



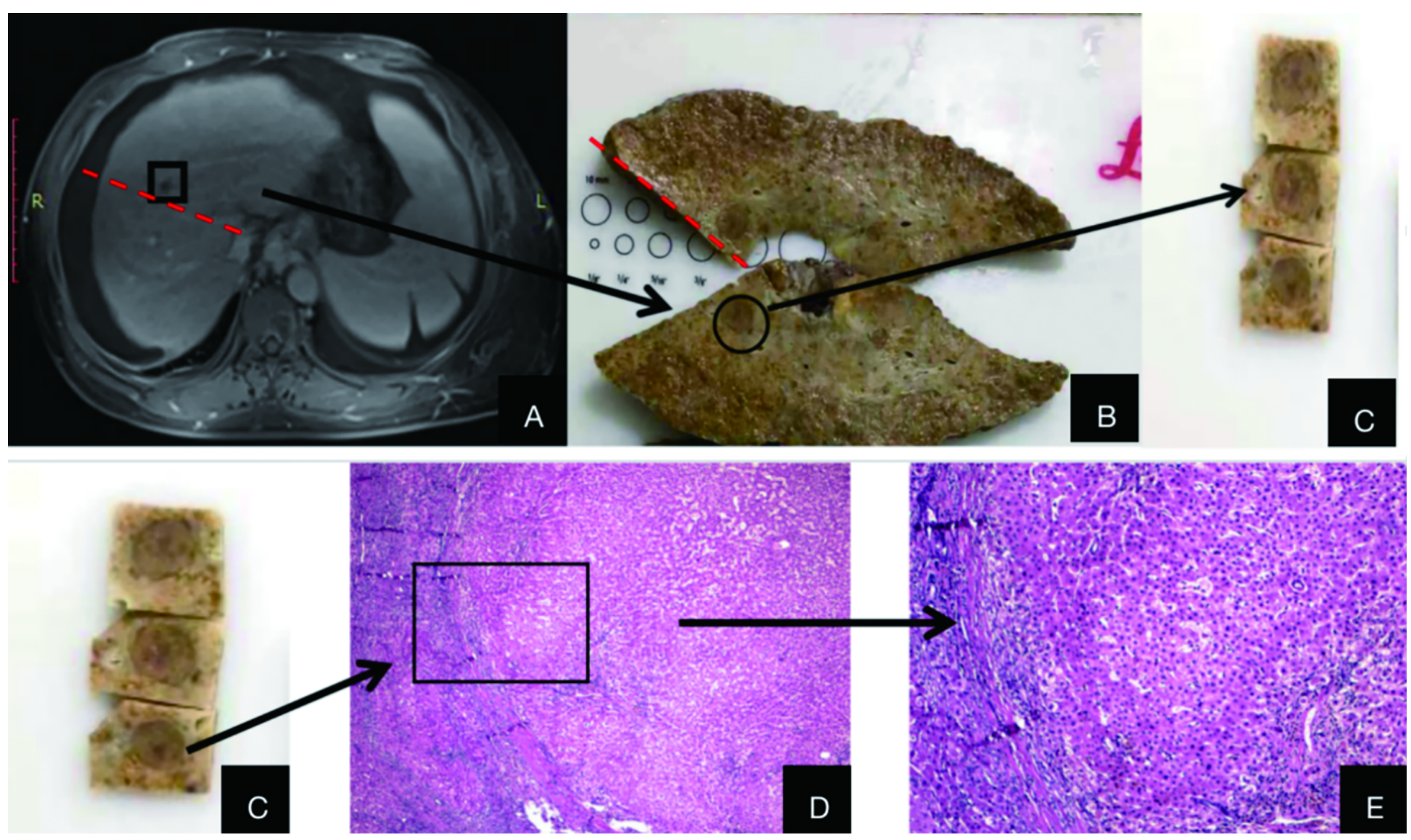

\section{Figure 1}

Images from a middle-aged male patient underwent liver transplantation, with a high-grade dysplastic nodule (HGDN) confirmed by pathology. Fig. A: lesion located in segment $\triangle a$ (black square). Fig. $B$ and C: gross specimen of liver and lesion. Fig. D: HE × 4. Fig. E: HE × 10 . 

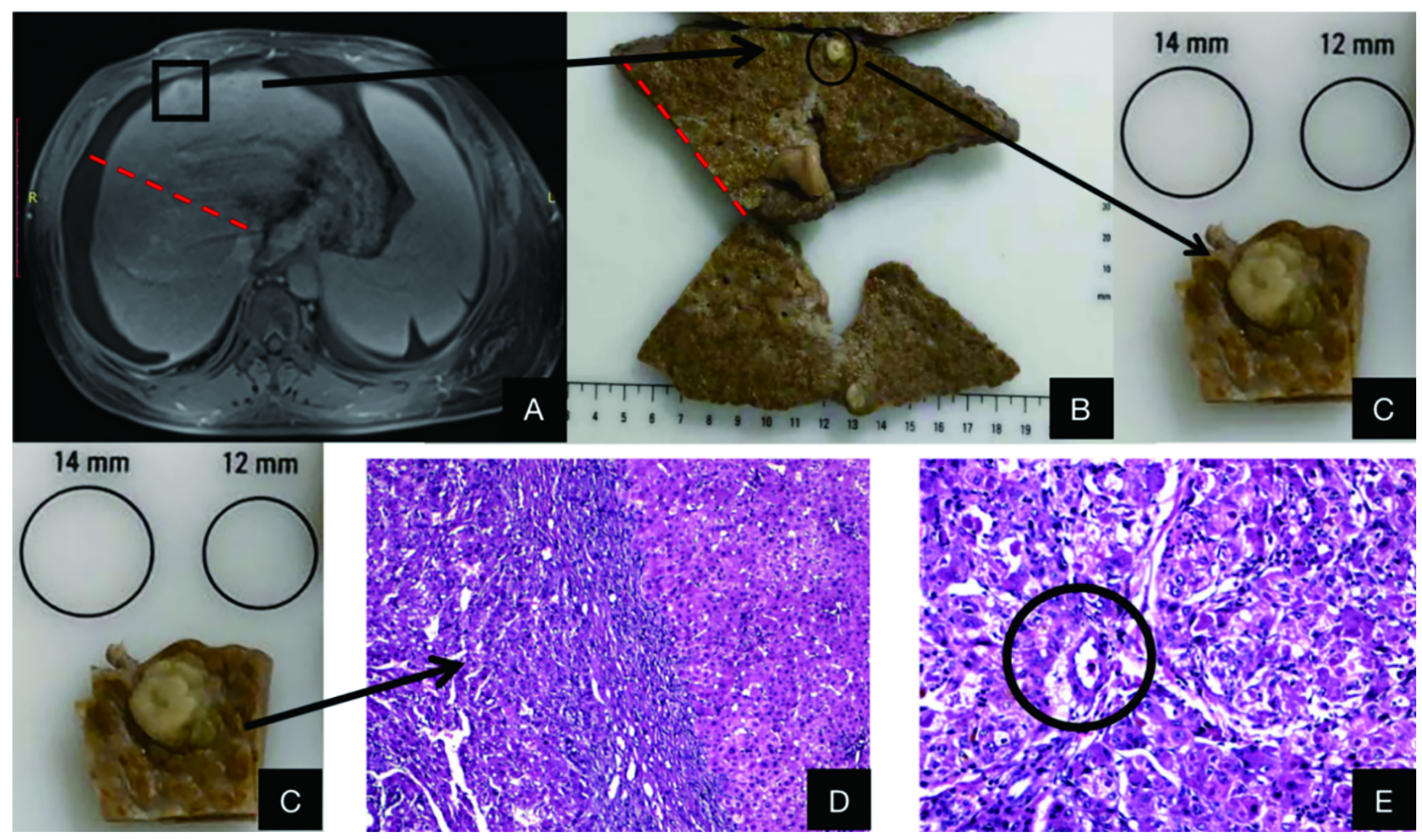

Figure 2

Images from the same patient, with moderately differentiated s-HCC confirmed by pathology. Fig. A: lesion located in segment $\triangle a$ (indicated with black square). Fig. B and C: gross specimen of liver and lesion. Fig. D: HE × 10. Fig. E: Unpaired arteries in liver cancer lesion (black circle). 

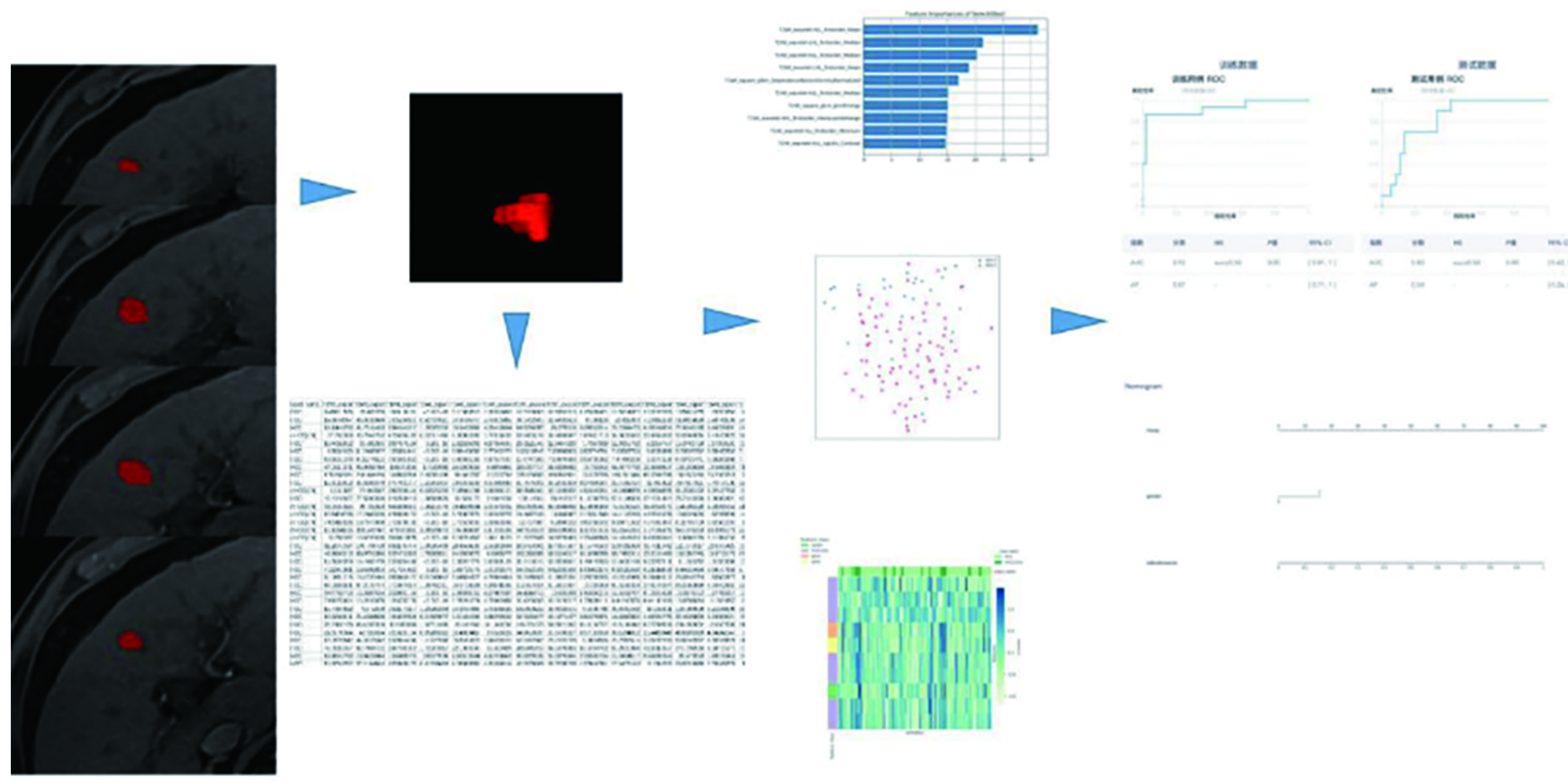

\section{Figure 3}

Flow chart of radiomics model establishment
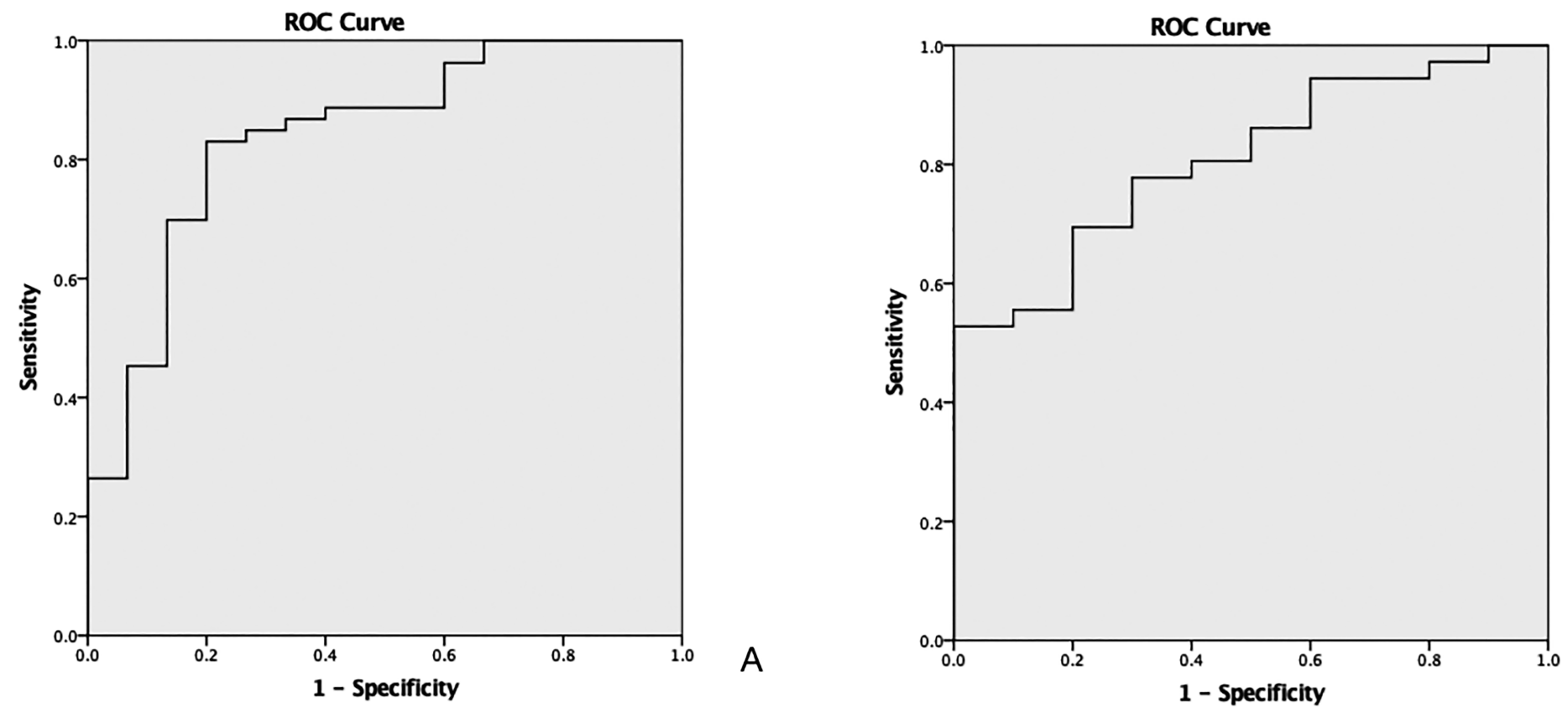

B

Figure 4

ROCs of radiomics model based on T1WI images (A: ROC of training group; $\mathrm{B}$ : ROC of test group) 

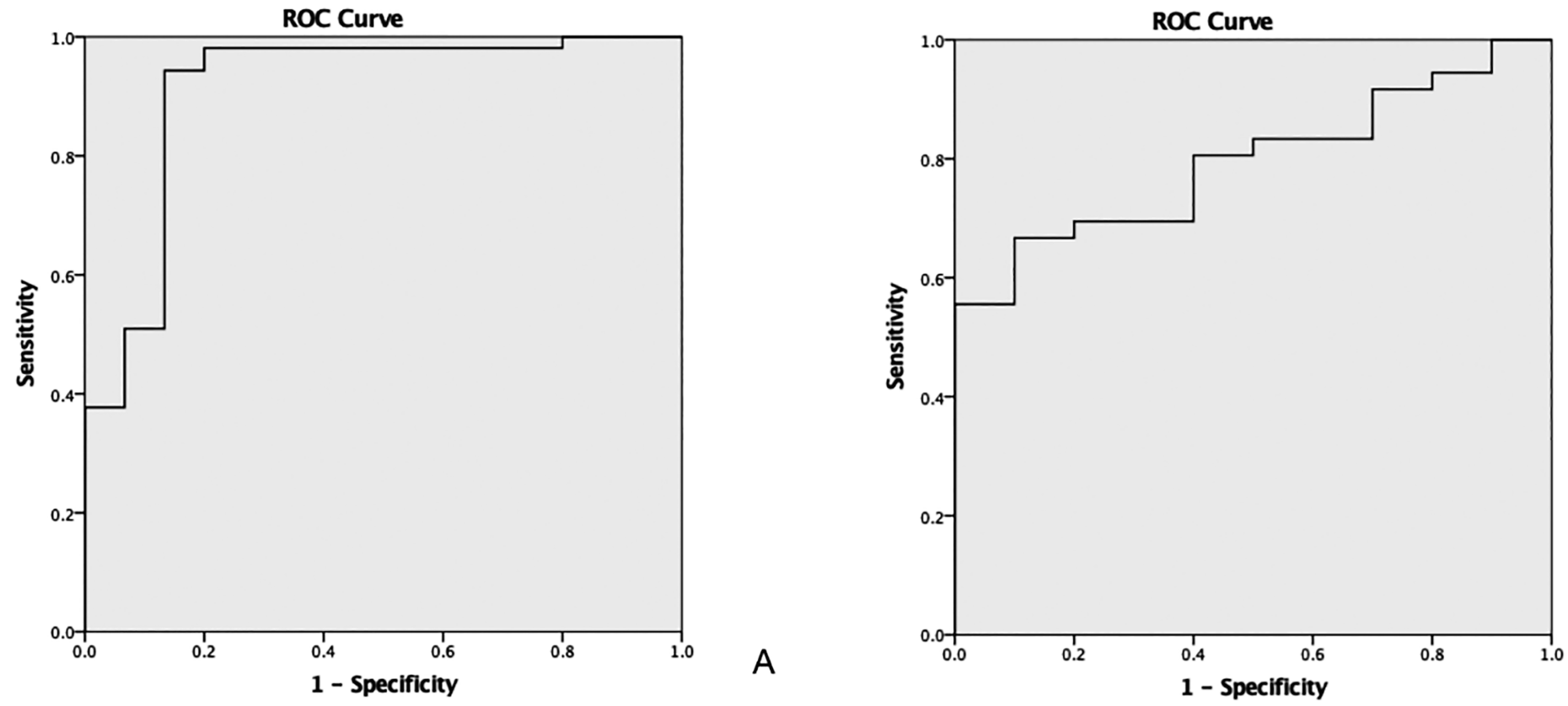

B

Figure 5

ROCs of radiomics model based on T2WI images (A: ROC of training group; $\mathrm{B}$ : ROC of test group)
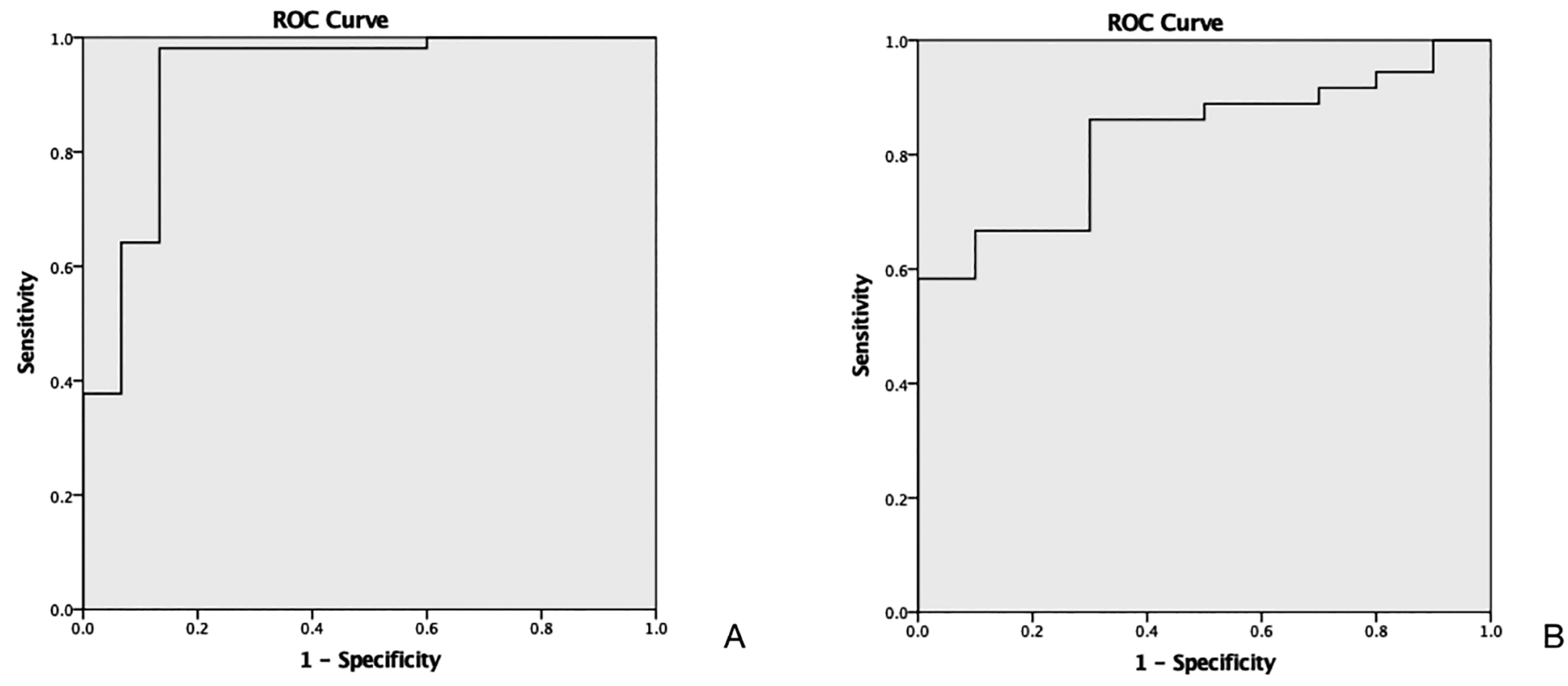

Figure 6

ROCs of radiomics model based on $\mathrm{T} 1 \mathrm{WI}+\mathrm{T} 2 \mathrm{WI}$ images (A: ROC of training group; $\mathrm{B}$ : ROC of test group) 


$\begin{array}{lllllllll}0.01 & 0.05 & 0.1 & 0.3 & 0.5 & 0.7 & 0.9 & 0.95 & 0.99\end{array}$

\section{Figure 7}

Nomogram obtained by the fusion model (gender 0 denotes male) 

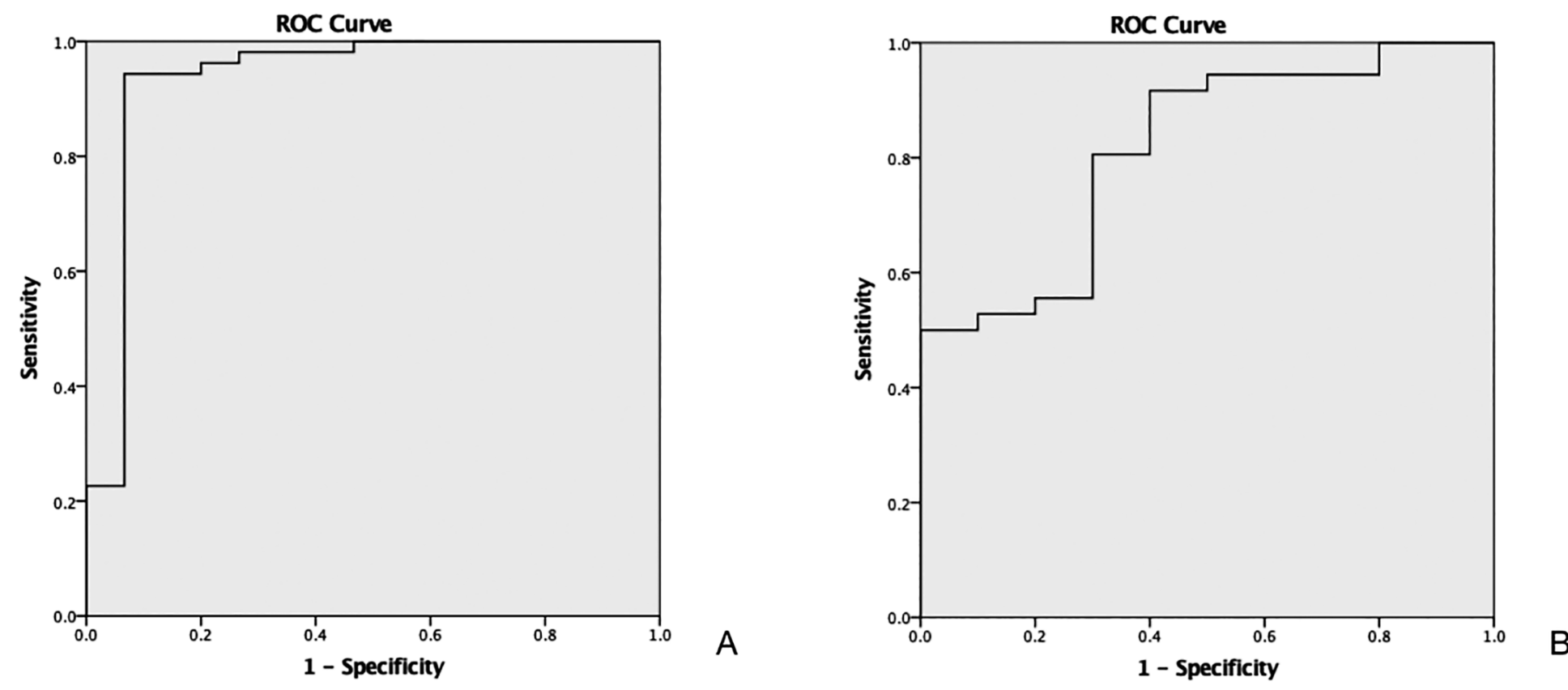

Figure 8

ROC of fusion model (A: ROC of training group; B: ROC of test group)

\section{Supplementary Files}

This is a list of supplementary files associated with this preprint. Click to download.

- Supplementary.docx 